\title{
ACTINOMYCES NAESLUNDII, A PREVIOUSLY UNDOCUMENTED CAUSE OF INFECTIVE ENDOCARDITIS; WITH LITERARY REVIEW
}

\author{
${ }^{1}$ Loren Garrison Morgan, ${ }^{2}$ Ashley L. Davis, ${ }^{3}$ Paul Poommipanit and ${ }^{4}$ Yasir Ahmed \\ ${ }^{1,3}$ Department of Internal Medicine, Section of Cardiology, \\ Medical College of Georgia at Georgia Regents University, USA \\ ${ }^{2}$ Department of Emergency Medicine, School of Medicine, \\ University of South Carolina, Columbia, SC, USA \\ ${ }^{4}$ Department of Internal Medicine, Section of Infectious Diseases, \\ Texas Tech University Health Science Center, Odessa, TX, USA
}

Received 2014-07-03; Revised 2014-08-06; Accepted 2014-10-27

\begin{abstract}
Actinomycotic endocarditis is a rare disease with only 18 documented cases to date. Most of these cases are sub-acute or chronic infections and usually lack the stigmata of classic endocarditis. This article reviews the details of previously published reports of endocarditis due to various species of actinomyces, along with consensus opinion on diagnosis and treatment. We also present our unique case of Actinomyces naeslundii infective endocarditis, the first case ever attributed to this organism in medical literature.
\end{abstract}

Keywords: Actinomyces Naeslundii, Actinomyces, Infective Endocarditis, Prosthetic Aortic Valve, Bacteremia, Echocardiogram, Epidemiology, Pathogenesis, Antibiotic Therapy

\section{INTRODUCTION}

Actinomyces is a genus of facultative, anaerobic, gram-positive non-acid fast rods found in the oral cavity, gastrointestinal tract and in the female genital tract (Warren, 1996). Actinomyces sp. most commonly cause indolent, locally invasive tissue disease of the oro-cervical region, chest, abdomen or pelvis. Cardiac infections usually result from local extension of pulmonary lesions and tend to spread from the pericardium towards the myocardium. Hematogenous spread is exceedingly rare (Dutton and Inclan, 1968). Since the last review of this entity, several actinomyces species have been reclassified or newly identified. Medical literature to date has not identified any cases of $A$. naeslundi prosthetic or native valve Infective Endocarditis (IE).

\subsection{Case}

A 67 year old man who had undergone bovine prosthetic valve replacement in 2001 for bicuspid aortic valve stenosis and pacemaker placement in 2008 due to type II second degree AV block presented with worsening shortness of breath, fatigue, rigors, drenching sweats and a twenty pound weight loss over the last six weeks. He underwent routine dental cleaning one month before symptom onset. He had been admitted two weeks prior with mild dyspnea and fatigue but no fever. He underwent treatment for a presumed Congestive Heart Failure (CHF) exacerbation and was discharged home. Workup during that admission revealed an elevated White Blood Count (WBC) of $12,900 / \mathrm{mm}^{3}$ with a left shift. Two blood cultures were obtained at that time, which eventually grew gram positive rods, later identified as

Corresponding Author: Loren Garrison Morgan, Department of Internal Medicine, Section of Cardiology,

Medical College of Georgia at Georgia Regents University, USA 
Actinomyces naeslundii. He was recalled for admission and further workup.

His current exam was notable for a $3 / 6$ mid systolic murmur, bibasilar crackles and pitting edema. Routine laboratory evaluation was notable only for a WBC count of $13,600 / \mathrm{mm}^{3}$. The patient underwent Transesophageal Echocardiogram (TEE), which showed an ejection fraction of $35 \%$, thickening of the bovine aortic valve leaflets, a peri-prosthetic abscess located between the left atrium and the aortic annulus (Fig. 1) and a significant peri-valvular leak (Fig. 2) without obvious vegetations on the pacemaker wires or other valves.

Despite the concerning findings on TEE the patient adamantly refused any surgical intervention and opted for medical management. Due to the patient's history of a non-anaphylactic serum reaction to penicillin, intravenous ceftriaxone was chosen. Cultures were redrawn and patient was started on a 6 week course of antibiotics for treatment of $A$. naesludii IE. The patient tolerated the treatment well over the next five days and was discharged home to complete his antibiotic course.

At outpatient follow-up a week later, the patient appeared ill and was found to be hypothermic and hypotensive. Laboratory results showed a worsening leukocytosis of $18,200 / \mathrm{mm}^{3}$, significant transaminitis, anion gap acidosis and marked renal dysfunction. His repeat cultures from 10 days prior had once again grown A. naeslundii. The patient was readmitted and shortly thereafter had a fatal arrhythmia and could not be resuscitated. In accordance with the family's wishes, an autopsy was not performed.

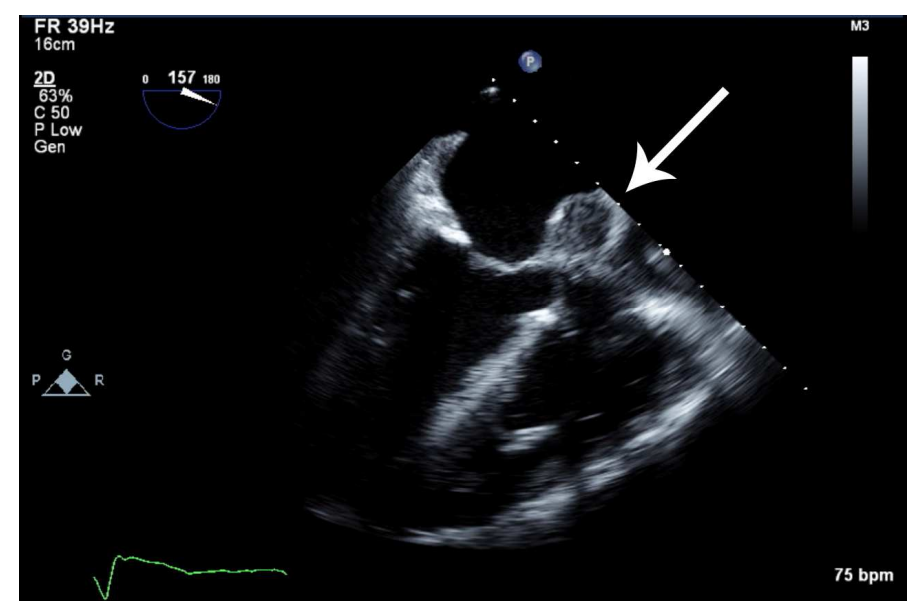

Fig. 1. TEE demonstrating thickening of the aortic valve leaflets and a confirmed perivalvular abscess pocket between the left atrium and the aortic annulus (arrow)

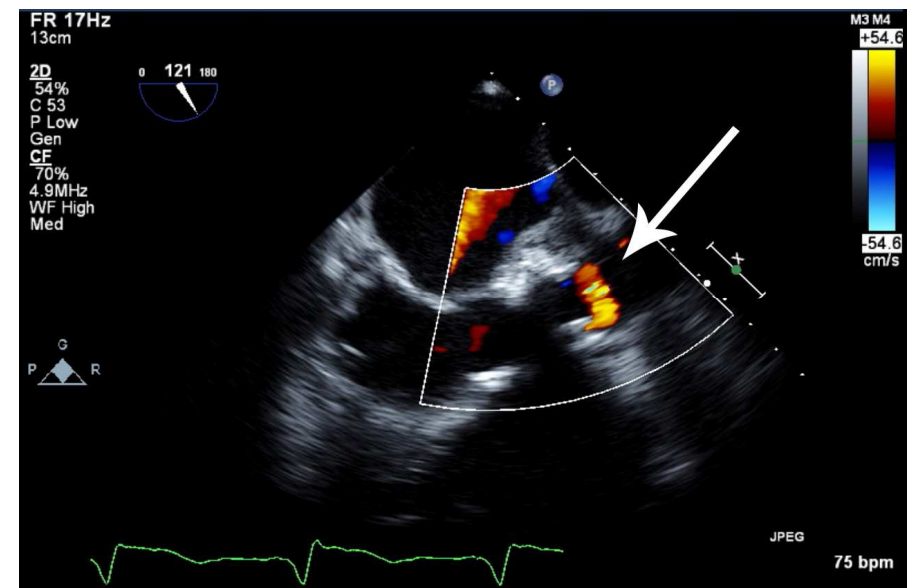

Fig. 2. TEE Doppler colorflow imaging demonstrating a significant periprosthetic valvular leak at the sight of the previously visualized abscess 


\section{DISCUSSION}

IE due to Actinomyces is rare, with only eighteen cases of primary actinomycotic endocarditis ever being reported in the literature. (Table 1) It should be noted when reviewing the literature that due to a nomenclature change, Actinomyces pyogene has been reclassified as Arcanobacterium pyogenes, thus lowering the total number of cases. Of the confirmed cases of Actinomyces IE, A. israelii, A. neuii, A. viscosus, A. funkei and A. meyeri have been documented in the literature. With the exception of one case, all of these cases were native valve endocarditis (Jitmuang, 2008; Grundmann et al.,
2010). None of the previously reported cases were ever attributed to $A$. naeslundii.

A. naeslundii is a microaerophilic, saprophytic inhabitant of the human oropharynx. There are several published cases of $A$. naeslundii causing infection outside the oral cavity, including infection of a penetrating leg injury, pelvic actinomycosis in the presence of an intrauterine device and infection of a prosthetic knee and hip joint; but none involving infection of either a native or a prosthetic cardiac valve (Vandevelde et al., 1995; Bonnez et al., 1985; Ruhe et al., 2001; Wüst et al., 2000).

Table 1. A Summary of 18 reported cases diagnosed primary actinomycotic endocarditis

\begin{tabular}{|c|c|c|c|c|c|c|c|c|c|}
\hline Ref & $\begin{array}{l}\text { Age } \\
\text { (year) }\end{array}$ & Sex & $\begin{array}{l}\text { Allied } \\
\text { cardiac } \\
\text { diseases }\end{array}$ & $\begin{array}{l}\text { Affected } \\
\text { valves }\end{array}$ & $\begin{array}{l}\text { Duration } \\
\text { of illness }\end{array}$ & Organism & Therapy & Complications & Result \\
\hline 3 & 38 & M & None & Mitral & 21 days & A. viscosus & Multiple $^{\mathrm{a}}$ & Cutaneous emboli & Alive \\
\hline \multirow[t]{2}{*}{3,4} & 37 & M & None & Mitral & 13 months & Actino sp. ${ }^{b}$ & Sulfa & Renal/GI emboli, CHF & Dead \\
\hline & 71 & $\mathrm{~F}$ & RHD & Aortic & 2 months & Actinos sp. ${ }^{b}$ & None & Renal failure & Dead \\
\hline 4 & 24 & M & None & Aortic/Mitral & 35 days & A. bovis & None & $\begin{array}{l}\text { CNS emboli, } \\
\text { CHF }\end{array}$ & Dead \\
\hline 4 & 55 & M & None & Aortic/Mitral & 9 months & Actino sp. ${ }^{b}$ & None & $\begin{array}{l}\text { CBS/GI/renal } \\
\text { emboli Dead }\end{array}$ & \\
\hline 4 & 39 & M & Murmur & Mitral & 42 days & Actino sp. ${ }^{\mathrm{b}}$ & Penicillin G & CNS emboli & Alive \\
\hline 4 & 43 & $\mathrm{~F}$ & RHD & Aortic/Mitral & 2 months & A. bovis & Penicillin G & GI emboli & Alive \\
\hline 4 & 6 & M & None & Mitral & 6 days & A. israelii & Penicillin G & $\mathrm{CHF}$ & Dead \\
\hline 4 & 70 & M & None & Mitral & 5 months & A. viscsus & Penicillin G & CNS emboli, CHF & Alive \\
\hline 4 & 65 & M & RHD & Aortic/Mitral & 28 days & A. israelii & Penicillin G & None & Alive \\
\hline 5 & 55 & $\mathrm{~F}$ & None & Mitral & 21 days & A. meyeri & $\begin{array}{l}\text { Ampicillin/ } \\
\text { Sulbactam }\end{array}$ & None & Alive \\
\hline 6 & 64 & M & AS & Aortic & 49 days & A. pyogene & Multiple $^{\mathrm{a}}$ & CNS emboli, CHF & Dead \\
\hline 7 & 81 & M & None & Aortic & NA & A. viscosus & $\begin{array}{l}\text { ceftizoxime } \\
\text { then ceftriaxone }\end{array}$ & None Alive & \\
\hline 8 & 40 & $\mathrm{~F}$ & $\begin{array}{l}\text { IVDU + } \\
\text { Previous IE }\end{array}$ & Tricuspid & 16 days & A. funkei & Multiple $^{\mathrm{a}}$ & Pulmonary emboli & Alive \\
\hline 9 & 43 & $\mathrm{~F}$ & Bicuspid AV & Aortic & 60 days & A. viscosus & $\begin{array}{l}\text { Multiple }^{\mathrm{a}} \\
\text { and AVR }\end{array}$ & $\mathrm{CHF}$ & Alive \\
\hline 10 & 68 & M & Bicuspid AV & Aortic & 21 days & A. neuii & $\begin{array}{l}\text { Multiple }^{\mathrm{a}} \\
\text { and AVR }\end{array}$ & Paravalvular abscess & Alive \\
\hline 11 & 46 & M & None & Mitral & 36 days & A. georgiae & Multiple $^{\mathrm{a}}$ & $\mathrm{CHF}$ & Alive \\
\hline 12 & 66 & M & AR s/p PV & Aortic & 2 months & A. neuii & Multiple $^{\mathrm{a}}$ & Paravalvular abscess & Alive \\
\hline PR & 67 & M & $\begin{array}{l}\text { Bicuspid AV } \\
\text { s/p PV + PM }\end{array}$ & Aortic & 2 months & A. naeslundii & Ceftraixone & Paravalvular abscess & Dead \\
\hline
\end{tabular}

Ref $=$ Reference, $\mathrm{M}=$ Male, $\mathrm{F}=$ Female, RHD = Rheumatic Heart Disease, AS = Aortic Stenosis, IVDU = Intravenous Drug User, IE = Infective Endocarditis, AV = Aortic Valve, AVR = Aortic Valve Replacement, CNS = Central Nervous System, GI $=$ Gastrointestinal, $\mathrm{CHF}=$ Congestive Heart Failure, $\mathrm{PV}=$ Prosthetic Valve, $\mathrm{PR}=$ Present Report, $\mathrm{PM}=$ Pacemaker; ${ }^{\mathrm{a}} \mathrm{At}$ least 2 antimicrobial agents including penicillin, ampicillin, amoxicillin-clavulanate, cephalosporins, gentamicin, vancomycin, clindamycin, rifampin or azithromycin were identified in the literature wheter empirically, combined or subsequently used; ${ }^{\mathrm{b}}$ Original classification no longer acceptable; there are insufficient data to determine these species; ${ }^{\mathrm{c}}$ Currently reclassified into Arcanobacterium pyogenes 
Like the present case, previous reports were described as sub-acute to chronic infections. The majority of the patients had no history of dental procedures and they did not show any classic stigmata of endocarditis. Fifty-three percent of the patients were complicated by systemic embolization to different organs including central nervous system, kidneys and gastrointestinal tract with a preponderance of the central nervous system (Jitmuang, 2008).

As can be seen by the scarcity of documented A. naeslundii infections in general, it is a rare diagnosis. This may be in part due to the difficulties encountered in microbiologic recovery and identification of the organism (Julian et al., 2005). In the previously discussed cases, isolation of the organism from blood cultures ranged from 2 to 7 days (median $=72 \mathrm{~h}$ ), but A. naeslundii can require ten or more days to grow in culture media, as noted in our case. An environmental reservoir has yet to be identified. To improve yield, the specimens should be collected with minimal exposure to oxygen and $\mathrm{CO}_{2}$ enriched environments and should be held up to 4 weeks (Julian et al., 2005; Tunkel and Kaye, 1992).

Actinomyces species are generally highly susceptible to beta-lactam antibiotics. Penicillins or cephalosporins are therefore considered first line agents. Alternatives include erythromycin, clindamycin, tetracyclines, or vancomycin, whereas gentamicin and ciprofloxacin show only limited activity (Funke et al., 1994). It is necessary to treat this disease with high doses, commonly as much as eighteen to twenty-four million units of the penicillin intravenously (IV) daily. Due to the small number of available cases, the optimal duration of therapy is debatable, but expert opinion favors long duration treatment of up to a year with oral antibiotics after the usual six week course of an IV beta-lactam (Grundmann et al., 2010). Prosthetic valve IE, particularly early prosthetic valve IE $(<$ twelve months after valve replacement), with perivalvular abscess and/or valve dehiscence typically requires surgical intervention. However, decisions regarding surgical intervention are complex and depend on many individual factors that vary among patients. These factors include: vegetation size, presence of perivalvular infection, evidence of embolism, heart failure, age and noncardiac morbidities (Baddour et al., 2005).

Of the case reports of actinomycotic endocarditis mentioned in this manuscript, there were quite a few cases in which survival was achievable merely by administration of prolonged antimicrobial therapy. Only two of these patients required surgical intervention, each of whom had evidence of worsening valvular regurgitation. In patients that did not respond to antimicrobial therapy, mortality was dramatically increased and almost inevitably led to death. However, due to innovations in both investigation and treatment, the mortality associated with primary actinomycotic endocarditis has decreased from $50 \%$ to $\sim 35 \%$ overall. Missed diagnosis and ineffective antibiotic treatment are the major causes of the death. The prognosis is good with early diagnosis, appropriate antimicrobial therapy and timely surgical intervention if needed (Jitmuang, 2008).

It is unclear if the patient's antecedent dental cleaning was related to the infection and whether antibiotic prophylaxis could have affected the course of illness. Current guidelines from the American Heart Association (AHA) do recommend antibiotic prophylaxis prior to dental procedures in patients with prosthetic valves. However, the AHA also states the evidence to support the effectiveness of endocarditis prophylaxis is not compelling and is likely to prevent only a tiny proportion of IE cases, at best (Wilson et al., 2007). This case, as well as previously reported cases, indicate that actinomycotic endocarditis is a potentially fatal disease with significant morbidity and mortality. While surgical intervention was certainly indicated and recommended in this case, the patients underlying co-morbidities made him a poor surgical candidate. It remains speculative if early operation would have changed the outcome in this case.

\section{CONCLUSION}

Prosthetic valve endocarditis due to Actinomyces is a rare condition but should be considered if the patient presents with constitutional symptoms such as weight loss, fatigue, along with worsening cardiac function or high clinical suspicion for IE. Besides holding the blood culture for at least ten days to allow proper time to isolate Actinomyces species, timely medical and surgical evaluation and treatment could improve the clinical outcome in such rare indolent infection.

\section{ADDITIONAL INFORMATION}

\subsection{Funding Information}

There was no funding for this manuscript.

\subsection{Author's Contributions}

Loren Morgan: Wrote the case report and conclusions, all work involved in submitting the manuscript. 
Ashley Davis: Wrote the discussion.

Paul Poommipanit: Major contributor to editing and finalization of manuscript.

Yasir Ahmed: Developed the complex figure summarizing multiple details of every previous case of actinomyces endocarditis. Provided infectious disease insight.

\subsection{Ethics}

There were no ethical issues for this manuscript.

\section{REFERENCES}

Baddour, L.M., W.R. Wilson, A.S. Bayer, V.G. Fowler and A.F. Bolger et al., 2005. Infective Endocarditis. Circulation, 111: e394-434. DOI: 10.1161/CIRCULATIONAHA.105.165563

Bonnez, W., G. Lattimer, N.A. Mohanraj and T.H. Johnson, 1985. Actinomyces naeslundii as an agent of pelvic actinomycosis in the presence of an intrauterine device. J. Clin. Microbiol., 21: 273-275.

Dutton, W.P. and A.P. Inclan, 1968. Cardiac Actinomycosis. Chest, 54: 65-67.

Funke, G., S. Stubbs, A. von Graevenitz and M.D. Collins, 1994. Assignment of human-derived CDC group 1 coryneform bacteria and CDC group like coryneform bacteria to the genus Actinomyces as Actinomyces neuii subsp. neuii sp. nov., subsp. nov. and Actinomyces neuii subsp. Anitratus subsp. nov. Int. J. Syst. Bacteriol., 44: 167-171. DOI: 10.1099/00207713-44-1-167

Grundmann, S., J. Huebner, J. Stuplich, A. Kock and K. Wu et al., 2010. Prosthetic valve endocartitis due to actinomyces neuii successfully treated with antibiotic therapy. J. Clin. Microbiol., 48: 10081011. DOI: 10.1128/JCM.01106-09
Jitmuang, A., 2008. Primary actinomycotic endocarditis: A case report and literature review. J. Med. Assoc. Thai.

Julian, K.G., L. De Flesco, L.E. Clarke and L.J. Parent, 2005. Actinomyces viscosus endocarditis requiring aortic valve replacement. J. Infect, 50: 359-362. DOI: 10.1016/j.jinf.2004.04.006

Ruhe, J., K. Holding and D. Mushatt, 2001. Infected total knee arthroplasty due to Actinomyces naeslundii. Scand. J. Infect. Dis., 33: 230-231. DOI: 10.1080/00365540151060987

Tunkel, A.R. and D. Kaye, 1992. Endocarditis with negative blood cultures. N Engl. J. Med., 326: 12151217. DOI: $10.1056 /$ NEJM199204303261809

Vandevelde, A.G., S.G. Jenkins and P.R. Hardy, 1995. Sclerosing osteomyelitis and Actinomyces naeslundii infection of surrounding tissues. Clin. Infect Dis., 20: 1037-1039. DOI: 10.1093/clinids/20.4.1037

Warren, N.G., 1996. Actinomycosis, nocardiosis and actinomycetoma. Dermatol. Clin., 14: 85-95. DOI: $10.1016 / \mathrm{S} 0733-8635(05) 70328-4$

Wilson, W., A.T. Kathryn, M. Gewitz, B.L. Peter and M.M. Larry, 2007. Prevention of Infective Endocarditis. Circulation, 116: 1736-1754. DOI: 10.1161/CIRCULATIONAHA.106.183095

Wüst, J., U. Steiger, H. Vuong and R. Zbinden, 2000. Infection of a hip prosthesis by Actinomyces naeslundii. J. Clin. Microbiol., 38: 929-930. 